University of Nebraska - Lincoln

DigitalCommons@University of Nebraska - Lincoln

Effects of pyriproxyfen and buprofezin on immature development and reproduction in the stable fly

Steven S. Xu

USDA-ARS, Steven.Xu@ars.usda.gov

Andrew Y. Li

USDA, ARS, Andrew.Li@ars.usda.gov

Kimberly Hutchinson Lohmeyer

USDA, ARS, kim.lohmeyer@ars.usda.gov

Adalberto A. Perez De Leon

USDA, ARS, beto.perezdeleon@ars.usda.gov

Follow this and additional works at: https://digitalcommons.unl.edu/usdaarsfacpub

Xu, Steven S.; Li, Andrew Y.; Lohmeyer, Kimberly Hutchinson; and Perez De Leon, Adalberto A., "Effects of pyriproxyfen and buprofezin on immature development and reproduction in the stable fly" (2012).

Publications from USDA-ARS / UNL Faculty. 1110.

https://digitalcommons.unl.edu/usdaarsfacpub/1110

This Article is brought to you for free and open access by the U.S. Department of Agriculture: Agricultural Research Service, Lincoln, Nebraska at DigitalCommons@University of Nebraska - Lincoln. It has been accepted for inclusion in Publications from USDA-ARS / UNL Faculty by an authorized administrator of DigitalCommons@University of Nebraska - Lincoln. 


\title{
Effects of pyriproxyfen and buprofezin on immature development and reproduction in the stable fly*
}

\author{
S. S. L I U, A. Y. LI, K. H. L O H MEYER and A. A. PÉ REZ DE LEÓN \\ USDA, ARS, Knipling-Bushland U.S. Livestock Insects Research Laboratory, Kerrville, TX, U.S.A.
}

\begin{abstract}
The stable fly, Stomoxys calcitrans (L.) (Diptera: Muscidae), is one of the most significant biting flies that affect cattle. The use of traditional insecticides for stable fly control has only a limited success owing to the insect's unique feeding behaviours and immature development sites. A laboratory study was conducted to evaluate the effects of two insect growth regulator (IGR) products, pyriproxyfen and buprofezin, on the development of the immature stages of the stable fly and the effects of pyriproxyfen on oviposition and egg hatch. Both pyriproxyfen and buprofezin had significant inhibitory effects on immature development. The $\mathrm{LC}_{50} \mathrm{~s}$ of pyriproxyfen and buprofezin were 0.002 and 18.92 p.p.m., respectively. Topical treatment of adult females with different doses of pyriproxyfen had significant negative effects on both female oviposition and egg hatching when 1- and 3-day-old females were treated, and the effects were dose dependent. A significant reduction in the mean number of eggs laid was observed only at the highest pyriproxyfen dose ( $8 \mu \mathrm{g} / \mathrm{fly})$ and egg hatch was unaffected by pyriproxyfen treatment when 5-day-old females were treated. Results from the present study indicate that pyriproxyfen has the potential to be used as part of an integrated stable fly management programme.
\end{abstract}

Key words. Stomoxys calcitrans, bioassay, egg hatch, insect growth regulator, larval development, oviposition, sub-lethal effect.

\section{Introduction}

The stable fly, Stomoxys calcitrans (L.), is one of the most significant biting fly pests that affect cattle, horses and dogs (Zumpt, 1973; Foil \& Hogsette, 1994). The stable fly has traditionally been a major pest affecting cattle in dairies and feedlots (Stork, 1979; Campbell et al., 1987), and it has more recently become a major pest of pastured cattle as a result of the increased use of round hay bales on pastures, the waste from which is an ideal habitat for stable fly larvae (Broce et al., 2005). Painful bites inflicted by stable flies can result in reduced weight gain and milk production, and in some cases contributes to reproductive failure (Berry et al., 1983; Campbell et al, 1987). The overall economic damage to the U.S. cattle industry is estimated at over 1 billion U.S. dollars (Taylor \& Berkebile, 2006).
Manure and green waste sanitation has been regarded as the primary method for stable fly control at confined cattle operations (Thomas et al., 1996), whereas the use of insecticides has only achieved limited success because of the unique feeding behaviours of the adult stable fly. It is difficult to apply insecticide to the lower parts of the animal, particularly legs, where stable flies prefer to feed. Secondary exposure of stable flies to insecticides that were used to primarily control the horn fly, Haematobia irritans irritans (L.), and the house fly, Musca domestica (L.) (Diptera: Muscidae) on cattle or throughout a premise contributed to the development of insecticide resistance in field populations of the stable fly (Cilek \& Greene, 1994; Marcon et al., 1997; Pitzer et al., 2010). Recent advances in stable fly control include treated targets, similar to those used for tsetse fly control, to which fastacting pyrethroids are applied (Foil \& Younger, 2006). Fairly

Correspondence: Dr Andrew Y. Li, USDA, ARS, Knipling-Bushland U.S. Livestock Insects Research Laboratory, 2700 Fredericksburg Road, Kerrville, TX 78028, U.S.A. Tel.: +1 830792 0341; Fax: +1 830792 0314; E-mail: Andrew.Li@ars.usda.gov

*This article reports the results of research only. Mention of a proprietary product does not constitute an endorsement or a recommendation by the USDA for its use. The USDA is an equal opportunity provider and employer. 
rapid accumulation of an allele that contributes to stable fly pyrethroid resistance development has been reported (Olafson et al., 2011), suggesting that it would be of interest to evaluate alternative insecticides for use on the treated targets.

Insect growth regulators (IGRs), including pyriproxyfen and buprofezin, are regarded as low-risk insecticides owing to their low toxicity in mammals and high selectivity against target pest species (Pener, 2002). Pyriproxyfen has been shown previously to be effective against several blood-feeding insects, including mosquitoes (Mulla et al., 1986; Iwanaga \& Kanda, 1988), sand flies (Mascari et al., 2010), triatomid bugs (Langley et al., 1990a), tsetse flies (Hargrove \& Langley, 1993; Langley et al., 1993) and horn flies (Bull \& Meola, 1993). There has been only one previously published study in the literature that reported the interaction of pyriproxyfen with the stable fly (Bull \& Meola, 1994), and no published work on the effectiveness of buprofezin against the stable fly. The objectives of the present study were to evaluate the effects of pyriproxyfen and buprofezin on immature development and adult emergence as well as determine the effects of pyriproxyfen on stable fly oviposition and egg hatch.

\section{Materials and methods}

\section{Flies}

Stable flies used in the present study were from a laboratory colony maintained at the USDA, ARS, Knipling-Bushland U.S. Livestock Insects Research Laboratory in Kerrville, TX, U.S.A. The fly colony was maintained under controlled conditions at $27.8 \pm 1{ }^{\circ} \mathrm{C}$ and $60 \pm 1 \% \mathrm{RH}$ with a LD 12; $12 \mathrm{~h}$ photoperiod regime. Adult flies were held in a screen cage with a solid bottom, and fed with bovine blood in cotton pads daily. The bovine blood contained $6.6 \mathrm{~g}$ of sodium citrate, $264 \mathrm{mg}$ of kanamycin sulfate and 264000 units of nystatin per liter. Larvae were reared on a Purina Fly Larva Media (PharmaServ, Framingham, MA, U.S.A.) covered with peanut hull pellets.

\section{Insecticides}

Pyriproxyfen (2-[1-methyl-2-(4-phenoxyphenoxy) ethoxy] pyridine)) (Nylar $\left.{ }^{\circledR} 10 \% \mathrm{w} / \mathrm{w}\right)$ was obtained from MGK Chemical Co. (Minneapolis, MN, U.S.A.). Buprofezin (2-tertbutylimino-5-phenyl-3-propan-2-yl-1,3,5-thiadiazinan-4-one) (40\% EC) was kindly provided by Nichino America Inc. (Wilmington, DE, U.S.A.).

\section{Manure (larval diet) bioassay}

To determine the effects of pyriproxyfen and buprofezin on stable fly larval development, a manure (larval diet) bioassay technique was used (Miller et al., 2003). The formulated pyriproxyfen and buprofezin were used to make serial dilutions in water. Eight pyriproxyfen concentrations $(0,0.001,0.005$, $0.01,0.05,0.1,0.5$ and 1 p.p.m.) and eight buprofezin concentrations $(0,5,10,25,50,75,100$ and 200 p.p.m.) were prepared in manure and each concentration was replicated three times. Next, $100 \mathrm{~g}$ of cow manure was added to a $500-\mathrm{mL}$ plastic drinking cup. A volume of $5 \mathrm{~mL}$ test solution was added to the manure in the cup and mixed thoroughly with a handheld spatula to generate the desired concentration of the IGR in the manure. Newly deposited stable fly eggs $(\sim 24 \mathrm{~h})$ were counted into groups of 100. A group of 100 eggs was added to the top of the treated manure. Each cup was then covered with a piece of tissue, and the tissue was secured with a rubber band. The experimental cups were placed in a flyrearing room and held at $25.6 \pm 1{ }^{\circ} \mathrm{C}$ and $55 \pm 1 \% \mathrm{RH}$ with a LD $12 ; 12 \mathrm{~h}$ photoperiod for 10 days. Pupae from each cup were then washed, counted and placed in a Petri dish for adult emergence. The number of adult males and females was counted after 5 days.

\section{Bioassays to determine the effects of pyriproxyfen on female oviposition and egg hatch}

To determine the effects of pyriproxyfen on female stable fly oviposition and egg-hatching, 5-day-old adult female flies were topically treated with six different doses of pyriproxyfen $(0.25,0.5,1,2,4$ and $8 \mu \mathrm{g} / \mu \mathrm{L})$ diluted in acetone, whereas flies treated with solvent alone were used as a control. Each experimental and control group comprised of 10 female and 10 male age-matched 5-day-old flies, of which only the females in the experimental groups were treated with pyriproxyfen.

Adult flies were knocked down briefly with $\mathrm{CO}_{2}$ before being placed on a cold table $\left(4^{\circ} \mathrm{C}\right)$ to be separated by sex. Cold immobilized flies were transferred to a counter top and each female in the group was treated by applying $0.5 \mu \mathrm{L}$ of pyriproxyfen solution (or acetone) to the dorsal surface of the thorax using a Hamilton repeating dispenser (Model no. PB600-1; Hamilton Company, WayReno, NV, U.S.A.). Each experimental dose was replicated three times. Each experimental group was transferred to a small cylindrical fly cage (10 $\mathrm{cm}$ in diameter $\times 4 \mathrm{~cm}$ high) and were fed once a day by placing a $7 \mathrm{~cm} \times 5 \mathrm{~cm}$ piece of blood-soaked absorbent pad (Maxim Hygiene Products, New York, NY, U.S.A.) on the top of the cage. Dead male and female flies were counted and removed from each cage daily.

Eggs were collected from the experimental and control groups by placing a piece of water-soaked absorbent pad wrapped in a black cloth on each test fly cage in the morning, daily, starting 6 days post-emergence. The pads were removed from the cages after $1 \mathrm{~h}$, and the number of eggs on each pad was counted. The absorbent pad with eggs from each cage was transferred into a plastic Petri dish with wet filter paper and left in the fly-rearing room for $48 \mathrm{~h}$ before eggs were examined to determine the egg hatching rate.

The effect of female fly age on its response to pyriproxyfen was evaluated by repeating the experiment using 3- or 1day-old flies separately. The experimental protocol on 3-dayold flies was exactly the same as for the 5-day-old flies. However, 1-day-old flies were highly sensitive to treatment with $\mathrm{CO}_{2}$, cold and acetone $(1.0 \mu \mathrm{L})$. As a consequence, a 
high percentage (50-70\%) of flies died during handling. The experimental protocol was therefore modified to minimize fly death from handling and treatment. Flies in a small group $(\sim 30$ flies at a time) were subject to $\mathrm{CO}_{2}$ and cold very briefly before topical treatment, and $0.5 \mu \mathrm{L}$ (instead of $1.0 \mu \mathrm{L}$ ) of test solution was applied to each female fly. The concentration of the test solutions was doubled $(0.5,1,2,4,8$ and $16 \mu \mathrm{g} / \mu \mathrm{L})$ in order to achieve the same topical dose of pyriproxyfen per fly that was used on the other two age groups of flies (3- and 5-day-old females).

\section{Data analysis}

POLO-PC (LeOra Software, 1987) was used to conduct probit analysis of the dosage response of the immature stage of the stable fly to manure treatment with the two IGRs, pyriproxyfen and buprofezin. To determine the effects of pyriproxyfen and buprofezin on stable fly development from egg to adult, the data were adjusted to correct for mortality in untreated controls (Abbott, 1925). Percentage inhibition of development was calculated as follows: $I=(1-N t / N c) \times$ 100 , where $I$ is the per cent inhibition of development; $N t$ is the number of adults that emerged from the treated group; and $N \mathrm{c}$ is the number of adults that emerged from the untreated control group. The mean responses among different treatment groups were statistically compared using the General Linear Models procedure (SAS Institute, 2004). Significant differences were accepted at $P=0.05$. Data were subjected to square root transformation in order to stabilize variances before SAS analysis.

\section{Results}

Effects of pyriproxyfen and buprofezin on the development of the immature stages

The development of the immature stages was significantly affected by pyriproxyfen treatment of manure (larval diet), and the effects were concentration dependent $(F=74.1$; d.f. $=7,8 ; P<0.001 ;$ Fig. 1 A). The pupation rate was significantly reduced at a pyriproxyfen concentration as low as 0.005 p.p.m. in manure (Fig. 1A). The treatment of manure with pyriproxyfen further significantly reduced the rate of adult emergence from the pupal stage $(F=88.6$; d.f. $=7,8$; $P<0.001$; Fig. 1C). No adults emerged at a pyriproxyfen concentration of 0.05 p.p.m. or higher (Fig. 1C). Compared with the untreated group, pyriproxyfen at concentrations of 0.005 and 0.01 p.p.m. caused $30.0 \%$ and $50.3 \%$ reductions in pupation rate. Additional $83.9 \%$ and $91.9 \%$ reductions in emergence of adults from pupae were also observed for the same treatment groups (Fig. 1A, C).

Buprofezin also had significant effects on the larval-to-pupal stage of development $(F=40.3$, d.f. $=7,22 ; P<0.001)$ and pupal-to-adult stage of development $(F=12.5$; d.f. $=12.5$; $P<0.001$; Fig. 1B, D); however, compared with that of pyriproxyfen, the effect of buprofezin was relatively weak. A significant effect of buprofezin on larva-to-pupa development was observed at 25 p.p.m., and the formation of pupae was completely blocked at 200 p.p.m. buprofezin. Similarly, a significant reduction in adult emergence from the pupal stage was also observed for buprofezin in the range of 75-200 p.p.m.
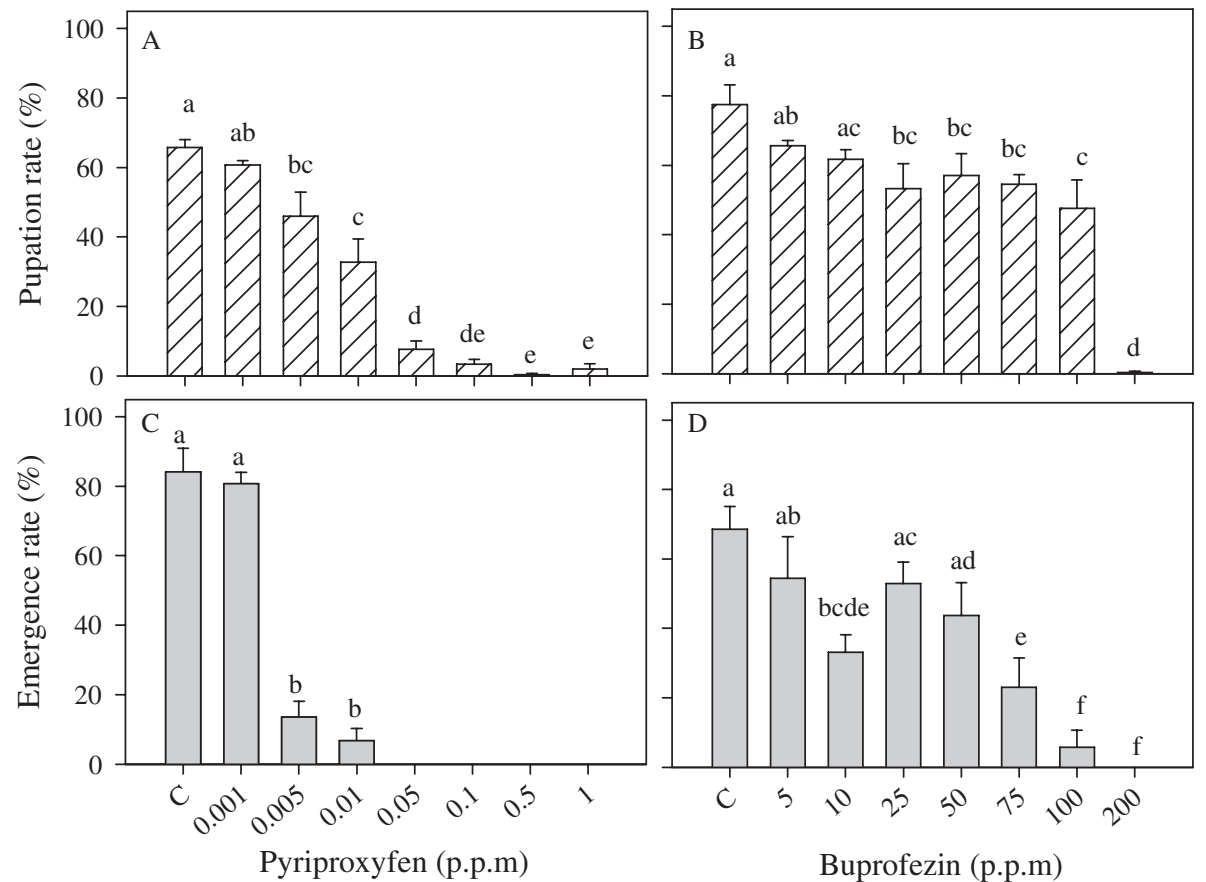

Fig. 1. Effects of pyriproxyfen and buprofezin on pupation rate (larval-to-pupal development, $A$ and $B$ ) and adult emergence rate (pupal-to-adult development, $\mathrm{C}$ and $\mathrm{D}$ ) in the stable fly, as assessed by treatment of the larval diet (manure). Means with different letters are significantly different (LSD test, $P<0.05$ ). 

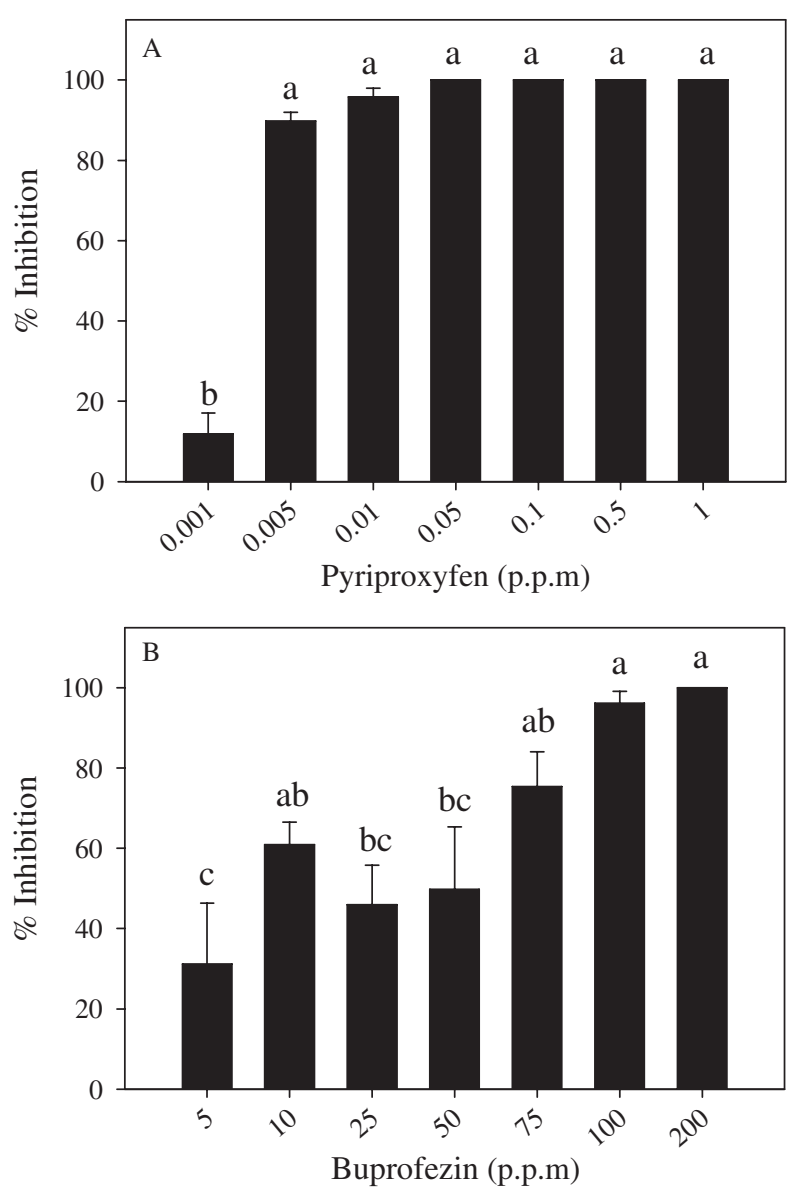

Fig. 2. Total per cent inhibition of the stable fly from egg to adult development as a result of treatment of the larval diet (manure) with different concentrations of pyriproxyfen (A) and buprofezin (B). Means with different letters are significantly different (LSD test, $P<0.05)$.

The overall inhibition of egg-to-adult development of the stable fly as a result of treating with manure using the two IGRs is shown in Fig. 2. Pyriproxyfen at as low as 0.005 p.p.m. in manure led to a $89.8 \%$ reduction in adult emergence and a $100 \%$ reduction was achieved at the concentration of 0.05 p.p.m. (Fig. 2A). Buprofezin caused a 31.1-49.8\% reduction in adult emergence at the concentration range of 5-50 p.p.m., with a total inhibition achieved only at 200 p.p.m. (Fig. 2B). The results of POLO-PC analysis of concentration responses of the stable fly to the two IGRs, evaluated as per cent inhibition of egg-to-adult development, are summarized in Table 1. The $\mathrm{LC}_{50}$ and $\mathrm{LC}_{90}$ of pyriproxyfen were 0.002 and 0.006 p.p.m., respectively, and the $\mathrm{LC}_{50}$ and $\mathrm{LC}_{90}$ of buprofezin were 18.9 and 187.5 p.p.m., respectively.

\section{Effects of pyriproxyfen treatment on female fly oviposition}

Female stable flies were topically treated with different doses of pyriproxyfen at 1,3 , or 5 days post-emergence. The mean numbers of eggs deposited per female during the first 5-day period in both the control (acetone only) and pyriproxyfentreated groups from all three age groups evaluated are shown in Fig. 3. In comparison to the control group, pyriproxyfen treatment of female flies had a significant effect on the mean number of eggs deposited per female for the test period for all age groups tested $(F \geq 4.6$; d.f. $=6,14 ; \quad P<0.0086)$, but a significant effect of pyriproxyfen on the oviposition rate of 5-day-old females was observed only at the highest pyriproxyfen dose ( $8 \mu \mathrm{g} / \mathrm{fly})$.

\section{Effect of pyriproxyfen on egg hatch of treated female flies}

Egg hatching rates for 1-, 3-, and 5-day-old females treated with varying concentrations of pyriproxyfen are presented in Fig. 4. A reduction in the egg hatch rate was observed in eggs laid by 1-day-old pyriproxyfen-treated females at a dose as low as $0.25 \mu \mathrm{g} /$ fly $(F=27.4$; d.f. $=6,14 ; P<0.0001)$. A similar dose-dependent decline in egg hatching was observed for the 3 -day-old female group ( $F=4.1$; d.f. $=6,11 ; P=0.0218$ ). No significant reduction in egg hatching $(F=2.4$; d.f. $=6,13$; $P=0.0929)$ was observed in 5-day-old pyriproxyfen-treated females.

\section{Discussion}

The results of the present study indicate that pyriproxyfen was highly effective against the immature stages of the stable fly when larvae developed in pyriproxyfen-treated manure, with an LC $_{50}$ of 0.002 p.p.m. or 2.0 p.p.b. Bull \& Meola (1993, 1994) previously reported the $\mathrm{LC}_{50 \mathrm{~s}}$ for pyriproxyfen against the immature stage of the horn fly (9.3 p.p.b.) and the stable fly (12.8 p.p.b.) using a similar manure bioassay. The pyriproxyfen $\mathrm{LC}_{50}$ (0.002 p.p.m. or 2 p.p.b.) for stable fly obtained

Table 1. Results of the probit analysis of dose responses of the stable fly to IGR treatment of manure (larval diet).

\begin{tabular}{lllllc}
\hline IGR & $n^{*}$ & Slope $\pm \mathrm{SE}$ & $\chi^{2}$ (d.f.) & $\mathrm{LC}_{50}(95 \% \mathrm{CI}) \dagger$ & $\mathrm{LC}_{90}(95 \% \mathrm{CI}) \ddagger$ \\
\hline Pyriproxyfen & 2600 & $2.98 \pm 0.29$ & $14.65(19)$ & $0.002(0.002-0.003)$ & $0.006(0.005-0.007)$ \\
Buprofezin & 3000 & $1.29 \pm 0.15$ & $238.49(23)$ & $18.92(1.59-39.47)$ & $187.48(96.89-1275.64)$ \\
\hline
\end{tabular}

$* n=$ total number of eggs used for manure bioassays.

$\dagger \mathrm{LC}_{50}$, lethal concentration (p.p.m.) that caused 50\% immature mortality.

$\ddagger \mathrm{LC}_{90}$, lethal concentration (p.p.m.) that caused $90 \%$ immature mortality.

IGR, insect growth regulator; CI, confidence interval. 

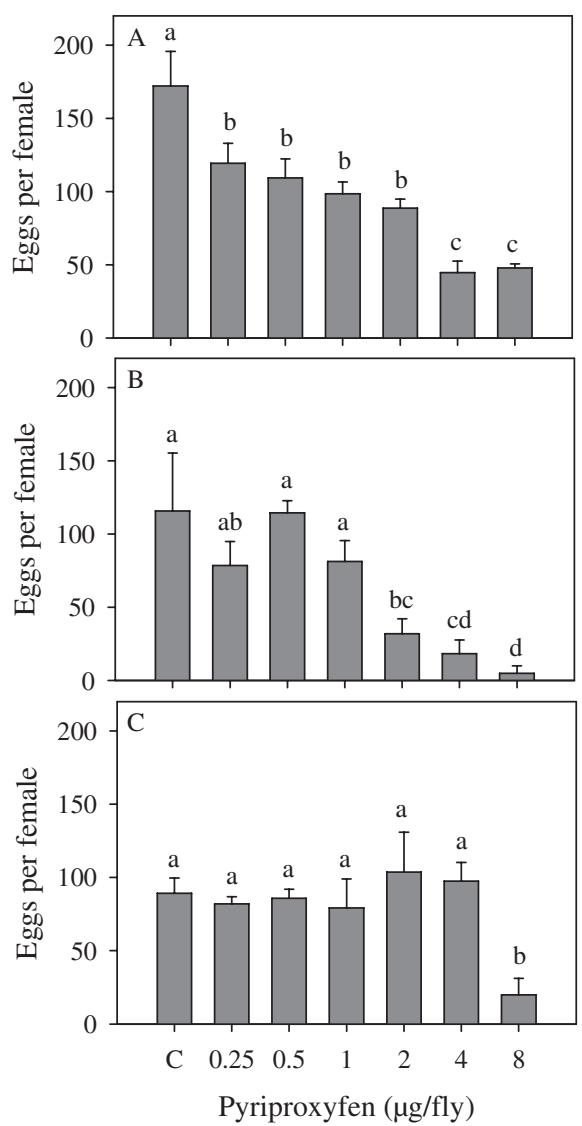

Fig. 3. Effect of topically applied pyriproxyfen on the oviposition of 1-, 3-, and 5-day-old female flies, as presented in (A), (B) and (C), respectively. Females were treated with different doses $(\mu \mathrm{g} / \mathrm{fly})$, and the mean number of eggs laid per female during the first 5 days of oviposition is presented. Means in the same panel with different letters are significantly different (LSD test, $P<0.05$ ).

from the present study was six-fold lower than previously reported by Bull \& Meola (1994). This could have been caused by differences in experimental conditions. Nevertheless, results from the present study suggest that pyriproxyfen can potentially be used for the control of immature stable flies, possibly using a feed-through formulation and other formulations and delivery methods.

As pyriproxyfen is a juvenile hormone analogue that inhibits insect egg hatching, molting of larvae and adult emergence (Ishaaya \& Horowitz, 1992), we measured the effects of pyriproxyfen on both pupation (larval-to-pupal development) and adult emergence. Pyriproxyfen was more effective in inhibiting adult emergence than preventing the formation of pupae. The previous studies by Bull \& Meola $(1993,1994)$ did not differentiate the effects on pupation and adult emergence of horn flies when pyriproxyfen-treated manure was tested for a reduction of adult emergence.

Although pupation and adult emergence were also significantly reduced at the higher concentrations of buprofezin in the present study, concentrations needed were considerably greater than the effective concentrations for pyriproxyfen. The
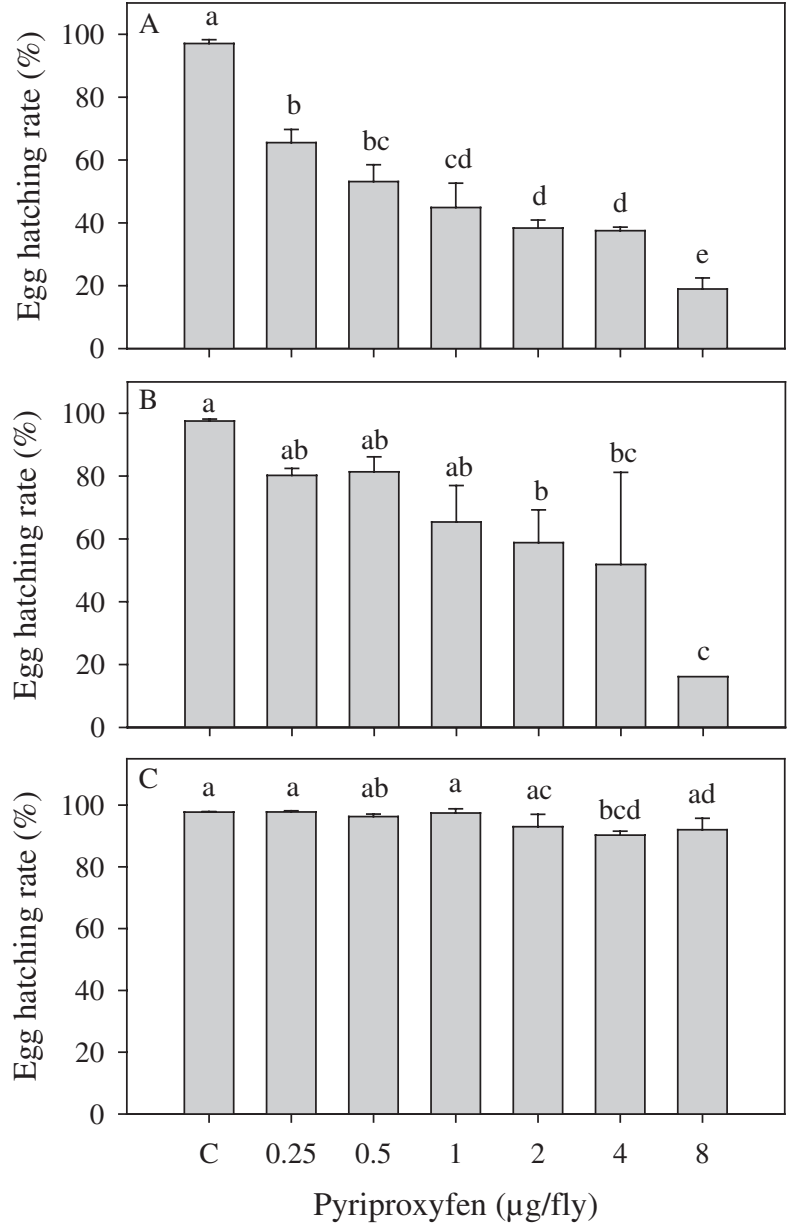

Fig. 4. Effect of topically applied pyriproxyfen on the egg hatch rate of 1-, 3- and 5-day-old female flies, as presented in (A), (B), and (C), respectively. Females were treated with different doses $(\mu \mathrm{g} / \mathrm{fly})$, and the hatching rate of eggs produced by the treated female flies during the first 5 days of oviposition is presented. Means in the same panel with different letters are significantly different (LSD test, $P<0.05$ ).

buprofezin $\mathrm{LC}_{50}$ (18.92 p.p.m.) was 9460 -fold higher than that of pyriproxyfen. As a result, much more buprofezin would be required to achieve the same level of immature control of pyriproxyfen when used to treat larval breeding sites.

Topical treatment of female flies with pyriproxyfen had a significant negative effect on both the mean number of eggs laid per female and the egg hatching rate; however, fly age played a role in the observed effects. Pyriproxyfen was effective in reducing both the number of eggs females produced and the egg hatching rate when 1-day-old female flies were treated at a dose as low as $0.25 \mu \mathrm{g} / \mathrm{fly}$. Pyriproxyfen did not cause significant reductions in egg number and hatching rate in 3 -day-old females at pyriproxyfen doses lower than $2.0 \mu \mathrm{g} /$ fly, and the treatment had no effect on 5-day-old females, that may have fully developed eggs, except a significant reduction in the number of eggs laid by females at a dose of $8.0 \mu \mathrm{g} / \mathrm{fly}$. The data suggests that pyriproxyfen may inhibit embryogenesis at the early stage of embryo development, leading to both 
reduced egg production and egg mortality after oviposition. This observation concurs with previous findings that treating freshly laid stable fly eggs with a pyriproxyfen solution did not have any adverse effects on egg hatch (Bull \& Meola, 1994).

Langley et al. (1990b) previously reported that a topical dose of $0.02 \mu \mathrm{g}$ of pyriproxyfen caused adult female tsetse flies to produce non-viable offspring for her entire life, a quantity that is 10 -fold less than the lowest dose effective against the stable fly. The difference could be attributed to varying sensitivities of the two fly species to this compound, or it may also result from different formulations used and locations of application. Langley et al. used rapeseed oil added to acetone solutions of pyriproxyfen at a $1: 4 \mathrm{v} / \mathrm{v}$ ratio, and $1 \mu \mathrm{L}$ of this solution was applied to the ventral abdomen of the female tsetse flies. The oil may facilitate pyriproxyfen penetration of the insect cuticle and direct application of pyriproxyfen to the ventral abdomen of the flies may also help pyriproxyfen directly reach the target organ (ovaries).

Results from the present study demonstrate the efficacy of pyriproxyfen against stable fly larvae at low concentrations $\left(\mathrm{LC}_{90}=6.0\right.$ p.p.b.) when larvae were exposed to treated manure. Therefore, pyriproxyfen has the potential to be used for treating stable fly larval habitats. Results of the topical application experiment also illustrate the detrimental effects of pyriproxyfen on oviposition and egg hatch, indicating that direct application to recently emerged female flies may be useful in reducing fecundity. Further studies are needed to evaluate the utility of pyriproxyfen as an IGR for the control of the immature stages and as a chemical sterilant for use on treated targets in an integrated stable fly control programme.

\section{Acknowledgements}

The authors would like to thank Drs Pia Olafson and Yu Cheng Zhu and two anonymous reviewers for critical review of the manuscript, Matthew Waldon for maintaining the stable fly colony and helping with larval diet bioassays, and Darci Burchers and DeEsta Hyatt for their assistance in bioassays. S.S.L. was supported by a USDA Postdoctoral Research Associate Program award to A.Y.L.

\section{References}

Abbott, W.S. (1925) A method of computing the effectiveness of an insecticide. Journal of Economic Entomology, 18, 265-267.

Berry, I.L., Stage, D.A. \& Campbell, J.B. (1983) Populations and economic impacts of stable flies on cattle. Transactions of the American Society of Agricultural Engineers, 26, 873-877.

Broce, A.B., Hogsette, J. \& Paisley, S. (2005) Winter feeding sites of hay in round bales as major developmental sites of Stomoxys calcitrans (Diptera: Muscidae) in pastures in spring and summer. Journal of Economic Entomology, 98, 2307-2312.

Bull, D.L. \& Meola, R.W. (1993) Effect and fate of the insect growth regulator pyriproxyfen after application to the horn fly (Diptera: Muscidae). Journal of Economic Entomology, 86, 1754-1760.

Bull, D.L. \& Meola, R.W. (1994) Interactions of the insect growth regulator pyriproxyfen with immature and adult stages of the stable fly. Southwestern Entomologist, 19, 257-263.
Campbell, J.B., Berry, I.L., Boxler, D.J., Davis, R.L., Clanton, D.C. \& Deutscher, G.H. (1987) Effects of stable flies (Diptera: Muscidae) on weight gain and feed efficiency of feedlot cattle. Journal of Economic Entomology, 80, 117-119.

Cilek, J.E. \& Greene, G.L. (1994) Stable fly (Diptera: Muscidae) insecticide resistance in Kansas cattle feedlot. Journal of Economic Entomology, 87, 275-279.

Foil, L.D. \& Hogsette, J.A. (1994) Biology and control of tabanids, stable flies and horn flies. Revue Scientifique et technique. Office International des Epizooties, 13, 1125-1158.

Foil, L.D. \& Younger, C.D. (2006) Development of treated targets for controlling stable flies (Diptera: Muscidae). Veterinary Parasitology, 137, 311-315.

Hargrove, J.W. \& Langley, P.A. (1993) A field trial of pyriproxyfentreated targets as an alternative method for controlling tsetse (Diptera: Glossinidae). Bulletin of Entomological Research, 83, 361-368.

Ishaaya, I. \& Horowitz, A.R. (1992) Novel phenoxy juvenile hormone analog (pyriproxyfen) supresses embryogenesis and adult emergence of sweetpotato whitefly. Journal of Economic Entomology, 85, 2113-2117.

Iwanaga, K. \& Kanda, T. (1988) The effects of a juvenile hormone active oxime ether compound on the metamorphosis and reproduction of an anopheline vector, Anopheles balabacensis (Diptera: Culicidae). Applied Entomology and Zoology, 23, 186-193.

Langley, P.A., Howl, V. \& Oouchi, H. (1990a) Regulation of reproduction in Rhodnius prolixus by the juvenile hormone mimic pyriproxyfen. Entomologia Experimentalis et Applicata, 57, 271-279.

Langley, P.A., Felton, T., Stafford, K. \& Oouchi, H. (1990b) Formulation of pyriproxyfen, a juvenile hormone mimic, for tsetse control. Medical and Veterinary Entomology, 4, 127-133.

Langley, P.A., Hargrove, J.W., Mauchamp, B. \& Oouchi, H. (1993) Prospects for using pyroproxyfen treated targets for tsetse control. Entomologia Experimentalis et Applicata, 66, 153-159.

LeOra Software (1987) POLO-PC: A User Guide to Probit or Logit Analysis. LeOra Software, Berkeley, CA.

Marcon, P.C.R.G., Thomas, G.D., Siegfried, B.D. \& Campbell, J.B. (1997) Susceptibility of stable flies (Diptera: Muscidae) from southern Nebraska beef cattle feedlots to selected insecticides and comparison of 3 bioassay techniques. Journal of Economic Entomology, 90, 293-298.

Mascari, T.M., Mitchell, M.A., Rowton, E.D. \& Foil, L.D. (2010) Evaluation of juvenile hormone analogues as rodent feed-through insecticides for control of immature phlebotomine sandflies. Medical and Veterinary Entomology, 14, 1-5.

Miller, A.J., Davey, R.B., Oehler, D.D., Pound, J.M. \& George, J.E. (2003) Efficacy of the Ivomec SR Bolus for control of horn flies (Diptera: Muscidae) on cattle in south Texas. Journal of Economic Entomology, 96, 1608-1611.

Mulla, M.S., Darwazeh, H.A., Kennedy, B. \& Dawson, D.M. (1986) Evaluation of insect growth regulators against mosquitoes with notes on nontarget organisms. Journal of the American Mosquito Control Association, 2, 314-320.

Olafson, P.U., Pitzer, J.B. \& Kaufman, P.E. (2011) Identification of a mutation associated with permethrin resistance in the para-type sodium channel of the stable fly (Diptera: Muscidae). Journal of Economic Entomology, 104, 250-257.

Pener, M.P. (2002) Insect growth regulators. Encyclopedia of Pest Management (ed. by D. Pimentel), pp. 398-401. Marcel Dekker, New York, NY. 
Pitzer, J.B., Kaufman, P.E. \& Tenbroeck, S.H. (2010) Assessing permethrin resistance in the stable fly (Diptera: Muscidae) in Florida by using laboratory selections and field evaluations. Journal of Economic Entomology, 103, 2258-2263.

SAS Institute (2004) The SAS for Windows, Version 9.1.3. SAS Institute, Cary, NC.

Stork, M.G. (1979) The epidemiological and economic importance of fly infestation of meat and milk producing animals in Europe. Veterinary Record, 105, 341-343.

Taylor, D.B. \& Berkebile, D. (2006) Comparative efficiency of six stable fly (Diptera: Muscidae) traps. Journal of Economic Entomology, 99, 1415-1419.
Thomas, G.D., Skoda, S.R., Berkebile, D.R. \& Campbell, J.B. (1996) Scheduled sanitation to reduce stable fly (Diptera: Muscidae) populations in beef cattle feedlots. Journal of Economic Entomology, 89, 411-414.

Zumpt, F. (1973) The Stomoxyine Biting Flies of the World. Gustav Fisher Verlag, Stuttgart.

Accepted 14 November 2011

First published online 12 March 2012 